\title{
CRIAÇÃO E TESTE DE UM MODELO PARA AVALIAÇÃO DE WEBSITES DE COMÉRCIO ELET'RÔNICO
}

\section{DEVELOPMENT AND TESTING OF AN E-COMMERCE WEBSITE EVALUATION MODEL}

Doutor em Ciências em Engenharia de Produção pelo Instituto Alberto Luiz Coimbra de Pós-graduação e Pesquisa da Universidade Federal do Rio de Janeiro (COPPE-UFRJ). Coordenador do Mestrado Executivo em Gestão Empresarial da Escola Brasileira de Administração Pública e de Empresas da Fundação Getulio Vargas (EBAPE-FGV). Rua Presidente Carlos de Campos, 1 15, bloco 502, Laranjeiras - Rio de Janeiro - RJ - CEP 22231-080 E-mail: luiz.joia@fgv.br

\section{LUIZ CLÁUDIO BARBOSA DE OLIVEIRA}

Mestre em Gestão Empresarial pela Escola Brasileira de Administração Pública e de Empresas da Fundação Getulio Vargas (EBAPE-FGV).

Pesquisador da EBAPE-FGV.

Praia do Botafogo, 190, sala 526, Botafogo - Rio de Janeiro - RJ - CEP 22250-900 E-mail: Icolive@openlink.com.br 


\section{RESUMO}

Este artigo visa desenvolver e testar, quantitativamente, um modelo de avaliação de websites de comércio eletrônico. O estudo tem, portanto, como objetivo a investigação das relações entre a interface de um website de comércio eletrônico e o comportamento do consumidor virtual, com ênfase em sua atitude e intenção de compra. O alvo deste trabalho é, assim, mensurar quais aspectos de uma loja virtual influenciam o usuário no seu processo de compra pela internet. Para realizar esse intento, adaptou-se o modelo de aceitação tecnológica (TAM) ao referencial teórico atual sobre o comportamento do consumidor na internet. O modelo gerado foi, então, testado e validado com base em um estudo empírico, usando-se a técnica de modelagem de equações estruturais (SEM). Para realização do teste de aceitação do modelo proposto, aplicou-se o modelo ao comércio varejista de CD, por ser este um dos principais artigos de venda nesse canal. O estudo conclui, para o comércio eletrônico de $\mathrm{CD}$, que a facilidade de uso, a confiança, e o prazer e a atratividade percebidos pelo usuário no website de venda de CD são aspectos-chave na compreensão de sua atitude e intenção de compra. Ao final, recomendações de práticas gerenciais associadas às conclusões da pesquisa e sugestões de estudos futuros são apresentadas.

\section{PALA VRAS-CHAVE}

Varejo eletrônico; Comércio eletrônico; Avaliação de websites de comércio eletrônico; Comportamento do consumidor na web; Modelo de aceitação tecnológica.

\section{ABSTRACT}

The scope of this research is to develop and test a model for evaluating $\mathrm{B}_{2} \mathrm{C}$ e-commerce websites quantitatively. Consequently, this study seeks to investigate the relationship between the website interface of $\mathrm{B}_{2} \mathrm{C}$ e-commerce and virtual customer behavior, emphasizing the purchasing attitude and intention. The 
objective of this paper is, therefore, to research which features of a virtual store effectively influence the user purchasing process via the Internet. In order to achieve this aim, the Technology Acceptance Model (TAM) was adapted for pertinent theoretical references about consumer behavior on the web. The model developed was then tested and validated through a Structural Equation Modeling (SEM) approach. An empirical analysis of the CD e-retailing industry in Brazil was then conducted, as this is one of the most important areas of the $\mathrm{B} 2 \mathrm{C} \mathrm{e}$-commerce realm. The study concludes that, for the electronic commerce of CDs, ease of use, trust, pleasure and attractiveness as perceived by visitors to CD e-retailing websites are key issues for understanding customer attitude and purchasing intention. Lastly, recommendations associated with the conclusions of this research are made, and further research options are proposed.

\section{KEYWORDS}

E-retailing; E-commerce; E-commerce website evaluation; Consumer behavior on the web; Tecnology acceptance model.

\section{INTRODUÇÃO}

Em um empreendimento virtual, a maior parte do contato com o cliente é feita por meio da interação deste com o website. A interface com o usuário atua como apresentação da empresa, mostruário, catálogo de produtos, vendedor, caixa, suporte, assistência técnica, serviços pós-venda etc. Todavia, a grande maioria dos websites não está preparada para tratar todos esses elementos, tendo dificuldade para conquistar a confiança dos usuários e para dar-lhes a necessária segurança para concretizar uma transação (ZHANG et al., 2000). Na verdade, alguns desses websites desestimulam a compra, quando deveriam ter o efeito contrário (NIELSEN, 200I).

Em paralelo, o varejo mundial percebe um crescimento em seu segmento on-line, representado mormente pela internet. Esse canal de distribuição vem aumentando sua participação no mercado varejista como um todo (PARENTE, 2000). Todavia, ainda não é completamente conhecido como esse novo ambiente afeta o comportamento do consumidor, isto é, suas atitudes e intenções no momento da decisão de compra. Para complementar esse cenário específico, vale ressaltar que quase toda a relação entre consumidor e empresa é realizada via website. Portanto, as características deste são responsáveis pelos elementos subjetivos e objetivos que irão influenciar a compra, sendo seu planejamento 
tarefa fundamental, que decidirá o sucesso ou fracasso do empreendimento virtual (NIELSEN, 200I).

O objetivo deste artigo é construir um modelo de avaliação de websites que permita identificar as características que contribuem para melhorar a atitude e intenção de compra dos consumidores virtuais. Esse modelo será aplicado ao segmento de varejo virtual de CD. Estudos similares de avaliação de websites de comércio eletrônico foram conduzidos por diversos autores (LOHSE; SPILER, I999; TURBAN; GEHRKE, I999; ZHANG et al., 2000; ZHANG; DRAN, 200I; SCHUBERT; SELZ, I999; LEDERED et al., 2000; HEIJDEN, 200I), porém cada um com objetivo e metodologia distintos.

Nesta pesquisa, buscaram-se adaptar os diferentes aspectos dos modelos já existentes, separando-se claramente os aspectos externos ao website - aqueles relacionados com as idiossincrasias do usuário - dos seus aspectos internos - aqueles relacionados ao seu design. Desse modo, procurou-se encontrar relacionamentos entre os diferentes fatores considerados, de modo a entender as suas contribuições para a efetivação de uma venda on-line.

$\mathrm{O}$ artigo está estruturado da seguinte forma: a primeira parte corresponde à fundamentação teórica e revisão bibliográfica do tema, com ênfase no modelo de aceitação tecnológica (TAM) adaptado à web, na classificação dos consumidores on-line e na importância da confiança percebida pelo usuário no ambiente digital. Com base nesse referencial teórico, apresentam-se a metodologia de estudo, as hipóteses de pesquisa e o modelo de análise da contribuição do website para o aumento da atitude e intenção de compra virtual. O modelo gerado é, então, testado, validado e comentado, por meio da sua aplicação ao varejo eletrônico de CD. Por fim, as contribuições da pesquisa, suas limitações e sugestões de caminhos para novos estudos são apresentadas.

\section{REVISÃO BIBLIOGRÁFICA}

\subsection{COMPORTAMENTO DO CONSUMIDOR NA WEB}

De acordo com Hoffman e Novak (I995), o relacionamento entre clientes e empresas vem se alterando com o tempo, por causa dos esforços de marketing para: buscar novas formas de resposta por parte dos consumidores, aumentar a penetração nos segmentos-alvo e aumentar o alcance de clientes. As organizações pretendem aumentar a influência de seu poder, sem perder a qualidade e a personalização do atendimento.

Para Brondmo (200I), os relacionamentos entre cliente e empresa atravessam um ciclo previsível de quatro estágios: atenção, consentimento, envolvimento e fidelidade. 
Para a obtenção da atenção do cliente, são necessárias ações promocionais que devem estar alinhadas ao planejamento de marketing da empresa e ser baseadas no tipo de relacionamento que se deseja ter com o cliente (BRONDMO, 200I).

A internet, ao facilitar o diálogo entre a empresa e o cliente, ressalta ainda mais a importância da obtenção de atenção. Isso se deve ao fato de que na web o cliente é quem escolhe qual site visitar, em meio a uma variedade praticamente inesgotável de opções (GODIN, 200I). Nela, o conteúdo do site deve ser suficientemente atraente para gerar visitas, ressaltando a importância de disponibilizar informações relevantes para os clientes (TURBAN et al., 2000).

Quando a empresa tiver a atenção do cliente, ela deve obter seu consentimento para a comunicação. Sua meta nesse estágio é atrair o interesse de seus clientes potenciais em estabelecer um relacionamento com a empresa (BRONDMO, 200I).

Nesse estágio, a iniciativa de contato comercial com o cliente parte da empresa, que remete conteúdo para ele, obtendo, antes, a sua autorização para o envio (TURBAN et al., 2000). Um exemplo de aplicação desse tipo de tecnologia é o e-mail, quando usado para envio de material publicitário. Para que esse tipo de publicidade seja mais efetivo, a mensagem deve ser de interesse do consumidor e ter sua prévia aprovação de envio. A solicitação da autorização do consumidor para envio de informações colabora para o estabelecimento de uma relação de confiança que o consumidor deseja e valoriza (HOFFMAN; BATESON, I999).

No processo de envolvimento do cliente, a diversidade de informações existentes sobre o produto torna-se relevante, na medida em que a internet é um meio que não estimula as compras por impulso (REEDY et al., 200I). O cliente precisa estar convencido da utilidade de um produto para querer adquiri-lo. Dessa forma, o fornecimento abundante de informações, a oferta de amostras do produto, como o primeiro capítulo de um livro, e a facilidade para localizar a informação são itens importantes na decisão de compra do consumidor (REEDY et al., 200I).

Para todos os clientes, a escolha é influenciada pelo valor, que é formado principalmente por percepções de qualidade, preço e conveniência para a compra (RUST et al., 200I).

Quando um cliente potencial efetua sua primeira compra, ele sai da posição de ouvinte e passa a ser um cliente de fato. Nessa fase, deve ser preparada a base de um relacionamento, por meio da oferta de informações, promoções e entretenimento, que combinem com o interesse do cliente. É por esse relacionamento que surge o envolvimento (BRONDMO, 200I).

Por fim, para passar do envolvimento para a fidelidade, deve haver um comprometimento entre as partes. Nessa etapa, informações sobre os clientes devem ser constantemente capturadas, armazenadas e analisadas, com o objetivo de 
aumentar o nível das comunicações individualizadas e a adequação dos produtos e serviços oferecidos aos clientes (BRONDMO, 200I). Assim, um dos indicadores da fidelidade do cliente é a freqüência com que este compra da mesma empresa.

Além disso, a taxa de conversão de visitantes em clientes passa a ser um ponto nevrálgico na construção do relacionamento de um website com seus usuários. Dessa forma, o entendimento do objetivo pelo qual os consumidores utilizam a internet e o seu próprio estilo de vida e predisposição de compra associados ao mundo digital são assuntos discutidos por diversos autores.

Moe e Fader (200I) identificaram dois comportamentos antagônicos na rede: procura exploratória ou por objetivo. Turban et al. (2000) denominaram esses comportamentos de hedonista ou utilitário. Wolfinbarger e Gilly (200I) chamaram essa conduta de objetiva ou baseada em experiências. Seja como for, é possível identificar dois perfis diferentes de internautas - fato importante na avaliação dos requisitos de um website.

Por sua vez, Bellman et al. (I999), em pesquisa sobre estilo de vida e perfil de compra, concluíram que existe um grupo de indicadores - associados a quão "conectados" (wired) estão os usuários - que permite avaliar a predisposição desses usuários para compra on-line. Segundo os autores, os indicadores demográficos não têm nenhuma influência sobre o comportamento de compra dos consumidores on-line. Uma vez dentro da internet, o maior indicador para a tendência à compra é a procura por informações sobre produtos e o tempo de uso da internet. Dessa forma, os autores concluíram que os maiores compradores da internet possuem um estilo de vida muito parecido, denominado wired style (BELLMAN et al., I999).

Kim et al. (2000) estenderam o conceito de predisposição para a compra on-line, incluindo o risco e o benefício percebido na compra como fatores primordiais em uma transação. Além disso, corroboraram as idéias de Bellman et al. (I999), na medida em que perceberam que consumidores com estilo orientado pela internet (wired) percebem maiores benefícios em uma compra on-line. Além disso, concluíram que consumidores mais orientados por tempo também percebem maiores benefícios em comprar bens e serviços on-line, o que vem de encontro ao comportamento utilitário-hedonista apresentado por Moe e Fader (200I), Wolfinbarger e Gilly (200I) e Turban et al. (2000).

\subsection{MODELO DE ACEITAÇÃO DA TECNOLOGIA NA WEB}

O modelo de aceitação da tecnologia (technology acceptance model) - em sua versão original - foi desenvolvido por Davis (I989). O objetivo era explicar o uso da tecnologia da informação pelos usuários, adotando uma relação causal baseada em: crenças e percepções, atitudes, intenções e comportamento. Segundo o modelo, 
esses conceitos estariam conectados por meio da "Teoria da ação racional" - criada por Fishbein e Ajzen (apud HEIJDEN, 2000a) -, que prega que as percepções de uma pessoa formam suas atitudes sobre um determinado objeto. Essas atitudes, por sua vez, definiriam as intenções, modificando o comportamento do usuário.

Esse modelo vem sendo aplicado por diversos autores como fonte de explicação da satisfação dos clientes com um determinado website (HEIJDEN, 200ob; LEDERED et al., 2000; VENKATESH, 2000). Cada pesquisa foi realizada com objetivos diferentes, criando distintas ênfases em cada um dos elementos e agregando/retirando novos componentes de explicação.

De acordo com modelo proposto por Heijden (2000b), a utilidade percebida e a facilidade de uso seriam os principais fatores a influenciar a atitude do usuário de um sistema. Esses fatores modificariam seu comportamento, gerando uma motivação positiva ou negativa. Esse tipo de conduta determinaria o uso final do sistema. O modelo adota a premissa de que todos os elementos são mensuráveis e relacionados positivamente, isto é, quanto maior for a utilidade percebida, maior será o uso do sistema, e vice-versa.

A utilidade percebida foi definida como a expectativa que uma pessoa tem sobre como um sistema particular de informação pode auxiliar sua performance. A facilidade de uso, por sua vez, seria o grau de esforço despendido pelo usuário para aprender e utilizar um sistema particular de informação. A atitude em relação ao uso é representada pela avaliação do usuário sobre seu desejo de empregar a tecnologia (LEDERED et al., 2000). Já o comportamento motivado para o uso seria a atitude posta em ação, isto é, a realização do desejo de empregar a tecnologia (HEIJDEN, 2000b).

Zhang et al. (2000) classificaram os atributos associados à aceitação de um website em fatores higiênicos e motivadores. Essa separação foi feita com base numa pesquisa amostral. Os autores identificaram quatro categorias eminentemente motivacionais: descontração, respostas cognitivas, credibilidade e aparência. Além disso, três categorias foram consideradas predominantemente higiênicas: privacidade e segurança, navegação e aspectos técnicos. Uma das conclusões interessantes desse trabalho foi identificar que, para aceitação de um website, certas características devem existir sempre (higiênicas), enquanto outras características da interface (motivacionais) contribuem mormente para a satisfação do usuário, como descontração e aparência. Esses fatores só terão seu papel destacado se os fatores higiênicos já estiverem presentes.

\subsection{A IMPORTÂNCIA DA CONFIANÇA NA WEB}

Geralmente, os consumidores compram de varejistas de marca reconhecida mesmo que estes não ofereçam o produto que procuram ao menor preço. Isso 
sugere que outros fatores, como confiança, podem ser muito importantes no comércio eletrônico (GREENWALD; KEPHART, I999). A confiança nas transações on-line está se tornando um fator-chave de sucesso das atividades na internet (TURBAN et al., 2000).

A importância da confiança no mercado virtual também pode ser percebida nas pesquisas de comportamento do consumidor na web. Em grande parte dos casos, a segurança deficiente e a falta de confiabilidade são apontadas como motivos pelos quais algumas pessoas não fazem compras on-line (HANSON, 2000).

$\mathrm{Na}$ medida em que a internet cria hábitos de compra em que clientes e vendedores estão espacialmente e, temporariamente, separados, as partes envolvidas mantêm-se anônimas durante uma transação. Como vendedores e clientes precisam avaliar a integridade e respeitabilidade da outra parte, a confiança é um fator de muita importância no comércio eletrônico (SMITH et al., I999).

Quando um consumidor compra um item em uma loja física, em geral a transação é feita simultaneamente - ele paga pela mercadoria e deve receber no mesmo momento. Na loja virtual, na maioria dos casos, existe uma diferença de tempo entre a consumação do pedido, o pagamento e o recebimento do produto. Provavelmente, o consumidor também nunca falará diretamente com algum funcionário da empresa (REEDY et al., 200I).

A falta da presença física e o anonimato inerentes ao meio fazem que as percepções dos clientes sobre as empresas sejam ainda mais importantes nos negócios pela internet (LOEBBECKE; HORNIG, 200I).

Hoffman et al. (I998) e Jarvenpaa et al. (2000), entre outros, enfrentaram o desafio de mensurar a relação entre a compra no meio digital e a confiança no website, encontrando uma relação causal bastante expressiva. Além disso, concluíram que a preocupação do usuário com a confiança e risco do website depende de suas caracterísiticas idiossincráticas, isto é, seu estilo e orientação no mundo digital.

\section{MODELO PROPOSTO}

O modelo proposto por esta pesquisa alia as características de segurança e confiança propostas por Hoffman et al. (I998) e Jarvenpaa et al. (2000) com as características apresentadas por modelos de avaliação do sucesso de websites de comércio eletrônico. Entre estes, foram usados o modelo de aceitação tecnológica (TAM), modificado para a web por Heijden (200I), e modelos de avaliação de design de websites desenvolvidos por Turban e Gehrke (I999), Turban et al. (2000) e Zhang et al. (2000). Para complementar o estudo, devem-se considerar as características do usuário e seu estilo de vida (BELLMAN et al., I999), assim 
como seu comportamento de procura e compra na internet (WOLFINBARGER; GILLY, 200I; MOE; FADER, 200I; TURBAN et al., 2000).

O modelo está baseado em correlações existentes entre aspectos inerentes aos usuários e, portanto, externos ao controle da interface gráfica, com os aspectos existentes no design do website e, portanto, sujeitos ao controle por parte do empreendimento virtual. Desse modo, estilo do usuário e orientação do comportamento do usuário são aspectos considerados externos à interface gráfica (antecedentes do modelo). Por sua vez, o design do website influencia a percepção do consumidor em relação a quatros fatores importantes: utilidade percebida, facilidade de uso, prazer e atratividade percebidos, e confiança e risco no momento da compra, considerados aspectos internos relativos ao design do website. Assim, a união desses aspectos determinaria as atitudes do usuário em relação ao consumo virtual, em uma determinada loja. As atitudes levariam, então, a um comportamento motivado para o consumo e, finalmente, à intenção de compra por parte do consumidor.

\subsection{ASPECTOS EXTERNOS}

No que tange aos aspectos externos relativos à transação comercial na web, os consumidores podem ser classificados com base em seus objetivos de navegação, sua procura de ofertas, estilos de vida etc. Eles possuem necessidades e desejos únicos, e se comportam de acordo com suas experiências anteriores de compra (KOTLER; ARMSTRONG, I999).

Dessa forma, com base no referencial teórico apresentado anteriormente, as hipóteses relativas aos aspectos externos do website, a serem testadas estatisticamente, são apresentadas a seguir, separadas por grupo.

No primeiro grupo, analisa-se o estilo do usuário (wired), isto é, quanto mais wired for o estilo do usuário (conectado), maior será a sua percepção no que se refere às características do design do website. Portanto, são formuladas as seguintes hipóteses:

- Hs: o estilo do usuário wired está positivamente relacionado à utilidade percebida em um website.

- H2: o estilo do usuário wired está positivamente relacionado à facilidade de uso percebida em um website.

- $\quad H_{3}$ : o estilo do usuário wired está positivamente relacionado ao prazer e à atratividade percebidos em um website.

- $\mathrm{H}_{4}$ : o estilo do usuário wired está positivamente relacionado à confiabilidade percebida em um website (menor risco para o usuário). 
No segundo grupo, estão formuladas as hipóteses relativas à orientação do usuário, isto é, quanto maior for a orientação do comportamento do usuário para a objetividade, maior será a sua percepção dos aspectos utilidade, facilidade de uso e prazer e atratividade do website. No entanto, a sua percepção de confiança no website será menor. Com base nisso, formulam-se as seguintes hipóteses:

- H5: a orientação do usuário por objetivos está positivamente relacionada à utilidade percebida em um website.

- H6: a orientação do usuário por objetivos está positivamente relacionada à facilidade de uso percebida em um website.

- H7: a orientação do usuário por objetivos está positivamente relacionada ao prazer e à atratividade percebidos em um website.

- H8: a orientação do usuário por objetivos está relacionada negativamente com a confiança percebida em um website.

Para mensuração dos fatores externos latentes "estilo do usuário" e "orientação do usuário", foram adotados os indicadores apresentados na Tabela I.

\section{TABELA I}

INDICADORES ASSOCIADOS AOS

\section{FATORES EXTERNOS DO MODELO}

\begin{tabular}{|c|c|}
\hline VARIÁVEL DO MODELO & INDICADORES \\
\hline \multirow{5}{*}{$\begin{array}{l}\text { Estilo do Usuário } \\
\text { (Wired) }\end{array}$} & Freqüência de compra de produtos na internet \\
\hline & Freqüência de procura de informações na internet \\
\hline & Tempo de uso da internet \\
\hline & Número diário de e-mails trocados \\
\hline & Tempo de disponibilidade da internet no trabalho \\
\hline \multirow{5}{*}{ Orientação do Usuário/Consumidor } & Uso da internet como diversão \\
\hline & Uso da internet para visitar websites específicos \\
\hline & Uso da internet para economia de tempo \\
\hline & Uso da internet com um objetivo definido \\
\hline & Procura de informações sobre produtos específicos \\
\hline
\end{tabular}

Fonte: Bellman et al. (I999), Moe e Fader (200I), Wolfinbarger e Gilly (200I) e Turban et al. (2000). 
- CRIAÇÃO E TESTE DE UM MODELO PARA AVALIAÇÃO DE WEBSITES DE COMÉRCIO ELETRÔNICO •

\subsection{ASPECTOS INTERNOS}

A análise dos fatores-chave de sucesso de um website varejista de CD é o principal objetivo deste estudo. Dessa forma, com base no referencial teórico apresentado anteriormente, formulam-se as seguintes hipóteses relativas aos aspectos internos do website, para que sejam testadas estatisticamente.

- H9: a utilidade e relevância da informação percebida estão relacionadas positivamente com a atitude e intenção de compra de CD no website.

- Hio: a facilidade de uso percebida está relacionada positivamente com a atitude e intenção de compra de CD no website.

- Hıs: o prazer e a atratividade percebidos estão relacionados positivamente com a atitude e intenção de compra de CD no website.

- Hı2: a confiança percebida está relacionada positivamente com a atitude e intenção de compra de CD no website.

Conforme foi demonstrado, o modelo combina/inclui certas características ao modelo de aceitação tecnológica (TAM) na web, facilitando o entendimento dos fatores críticos de sucesso da interface gráfica.

Para mensuração dos fatores internos latentes "utilidade percebida", "facilidade de uso percebida", "prazer e atratividade", "confiança e risco", e "atitude e intenção de compra”, adotaram-se os indicadores apresentados na Tabela 2.

\section{TABELA 2}

\section{INDICADORES ASSOCIADOS AOS}

FATORES INTERNOS DO MODELO

\begin{tabular}{ll}
\hline VARIÁVEL DO MODELO & INDICADORES \\
\hline \multirow{2}{*}{ Utilidade Percebida } & Conteúdo \\
\cline { 2 - 2 } & Velocidade da informação \\
\hline Facilidade de Uso Percebida & Navegação \\
\cline { 2 - 2 } & Organização \\
\cline { 2 - 2 } & Aspectos técnicos \\
\hline Prazer e Atratividade & Aparência \\
\hline
\end{tabular}

(continua) 


\section{TABELA 2 (CONCLUSÃO)}

INDICADORES ASSOCIADOS AOS

FATORES INTERNOS DO MODELO

\begin{tabular}{ll}
\hline VARIÁVEL DO MODELO & INDICADORES \\
\hline \multirow{2}{*}{ Confiança e Risco } & Privacidade \\
\cline { 2 - 2 } & Segurança das transações \\
\hline \multirow{2}{*}{ Atitude e Intenção de Compra } & Atitude \\
\cline { 2 - 2 } & Intenção de retorno \\
\hline & Intenção de compra \\
\hline
\end{tabular}

Fonte: Zhang et al. (2000), Heijden (200I), Hoffman e Novak (I998), Jarvenpaa et al. (2000), Turban et al. (2000) e Turban e Gehrke (I999).

Todos esses elementos, no entanto, devem ser testados com base na coleta de dados e avaliação da amostra. Somente após essa análise, será possível verificar se o modelo proposto adere às hipóteses pré-formuladas.

$\mathrm{Na}$ Figura I, o modelo proposto é representado, consolidadamente, por meio de seus relacionamentos associados às hipóteses apresentadas anteriormente.

\section{FIGURA I}



Fonte: Hoffman e Novak (I998), Jarvenpaa et al.(2000), Bellman et al. (I999), Moe e Fader (200I), Wolfinbarger e Gilly (200I), Turban et al. (2000), Turban e Gehrke (I999), Zhang et al. (2000) e Heijden (200I). 
- CRIAÇÃO E TESTE DE UM MODELO PARA AVALIAÇÃO DE WEBSITES DE COMÉRCIO ELETRÔNICO • LUIZ ANTONIO JOIA • LUIZ CLÁUDIO BARBOSA DE OLIVEIRA

\subsection{PROCEDIMENTOS DE AMOSTRAGEM}

A população deste estudo é composta de todos os brasileiros, consumidores de $\mathrm{CD}$, que utilizam a internet como canal de comercialização e/ou de informação sobre esse produto. O quadro de amostragem da pesquisa foi montado com base em grupos da internet, listas de discussão e outras fontes, chegando-se ao resultado apresentado na Tabela 3 .

TABELA 3

DADOS DO PROCESSO DE AMOSTRAGEM

\begin{tabular}{clc}
\hline PARÂMETRO & DESCRIÇÃO & VALOR \\
\hline$N$ & Número de casos no quadro amostral & 2.000 \\
\hline$N^{\prime}$ & Número de casos selecionados aleatoriamente & 1.500 \\
\hline$n$ & Número de casos na amostra & 496 \\
\hline$f$ & Fração amostral - taxa de resposta & $33,07 \%$ \\
\hline
\end{tabular}

Fonte: Trochim (200I).

No caso em questão, utilizou-se como padrão de resposta um formulário de preenchimento on-line. Um e-mail foi enviado para o usuário, estimulando a sua participação na pesquisa. Após a leitura do e-mail, um hiperlink direcionava o usuário para o website da pesquisa. Nesse processo, foi dispensado grande cuidado para evitar a idéia de mala-direta (ou spam), o que não corresponderia ao objetivo inicial. Como essa pesquisa lida com comércio eletrônico, via internet, não se considerou que a utilização deste canal para receber os dados causaria algum tipo de tendenciosidade na amostra. Afinal, todo comprador on-line é um internauta e, logo, poderia acessar a pesquisa. Um website foi criado para esse fim, coletando, entre maio e agosto de 2004, toda a informação necessária para a pesquisa, explicando os motivos do estudo e garantindo a privacidade do respondente e a seriedade do trabalho.

Nessa pesquisa, utilizou-se o método de amostragem aleatória simples proposto por Trochim (200I), baseado na lista obtida de possíveis respondentes. A utilização de questionário on-line, com validação das respostas por meio do uso de Javascript, evitou que o respondente deixasse qualquer questão em branco. O questionário foi elaborado de acordo com as recomendações de Taylor (I9992000) sobre E-survey, isto é, fácil e rápido de responder, e de modo que facilitasse o processo de tabulação dos dados. 
As técnicas utilizadas na análise dos dados possuem como premissa a normalidade e aleatoriedade da amostra. Para avaliação desses quesitos, foram realizados testes sobre desvios em relação à curtose (kurtosis) e à assimetria (skewness) da amostra. A avaliação da normalidade da amostra foi feita pelos testes de normalidade multivariada (SHARMA, I996) e pelo teste de aderência do qui-quadrado (goodness of fit) (SNEDECOR; COCHRAN, I989). Para análise da aleatoriedade da amostra, empregaram-se o run test e o teste da média quadrática das diferenças sucessivas propostos por Wonnacott e Wonnacott (1972). Em nenhum dos testes foi identificado algum indício de violação das premissas necessárias.

O questionário usou uma escala Likert de 5 pontos, e todas as variáveis, para efeito estatístico, foram consideradas ordinais. Nesse tipo de nível de mensuração, as variáveis guardam uma ordem bem definida, porém nada pode ser dito sobre as distâncias entre os atributos. A discussão sobre a escala utilizada é realizada no próximo tópico.

\subsection{MEDIDAS UTILIZADAS E COLETA DE DADOS}

Uma grande dificuldade para a coleta de dados em uma pesquisa é a criação/ formatação do questionário. Para evitar problemas de interpretação, um questionário foi distribuído inicialmente a um grupo piloto composto por quatorze pessoas. Esse grupo, além de responder ao questionário, pôde indicar pontos confusos e solicitar alteração na ordem das perguntas. Esse procedimento modificou bastante o formato original do formulário: as perguntas tornaram-se mais claras, eliminaram-se várias questões desnecessárias ou redundantes e foram solucionados problemas de escala, itens, ordenamento das questões e instruções de preenchimento. O questionário modificado foi, então, submetido a um préteste, com o mesmo grupo piloto, propiciando a realização de uma análise inicial dos dados. Dessa forma, tornou-se possível estimar o tamanho da amostra e verificar se as ferramentas estatísticas eram adequadas ao que se propunha.

O questionário final para a coleta de dados foi elaborado com base nas contribuições feitas pelo grupo piloto e pelos seguintes autores: Hoffman e Novak (I998), Jarvenpaa et al. (2000), Bellman et al. (I999), Moe e Fader (200I), Wolfinbarger e Gilly (200I), Turban e Gehrke (I999), Turban et al. (2000), Zhang et al. (2000) e Heijden (200I).

Como já dito anteriormente, um website foi criado para coleta dos dados e durante maio e agosto de 2004 foram recolhidos os questionários preenchidos pelos respondentes. 
- CRIAÇÃO E TESTE DE UM MODELO PARA AVALIAÇÃO DE WEBSITES DE COMÉRCIO ELETRÔNICO • LUIZ ANTONIO JOIA • LUIZ CLÁUDIO BARBOSA DE OLIVEIRA

\subsection{PROCESSO ESTATÍSTICO DE ANÁLISE}

Para análise dos resultados da pesquisa, empregaram-se métodos estatísticos e técnicas de análise multivariada. Os primeiros servem para analisar graficamente cada uma das variáveis, identificar outliers e confirmar premissas necessárias. As últimas servem para validar as hipóteses e o modelo proposto como um todo, bem como indicar o grau de relacionamento entre os constructos. Para esse propósito, foram utilizados os softwares estatísticos SAS 8.2 e LISREL 8.5I, que contribuíram para a análise fatorial e a modelagem de equações estruturais (structural equation model - SEM).

A aplicação do SEM permite muita flexibilidade à análise e pode ser utilizado para confirmar a existência de relação entre os constructos (confirmatory model strategy), possibilitando o uso de uma estrutura mais complexa de variáveis observadas - manifestas ou indicadores - e não observáveis - latentes ou constructos (HAIR JUNIOR et al., I998). O processo de análise inicia-se pela verificação do modelo de mensuração, pela avaliação do grau de confiabilidade das medidas e pela validação (convergente e discriminante) dos indicadores em relação aos contructos. Para tanto, foi utilizada a análise fatorial como instrumento de verificação da validade e confiabilidade dos constructos. Após essa análise, procede-se à investigação do modelo estrutural propriamente dito. Nesse ponto, é verificado o grau de aderência do modelo como um todo, bem como dos relacionamentos propostos entre as variáveis latentes.

\subsection{CARACTERIZAÇÃO DA AMOSTRA}

A amostra obtida foi comparada aos resultados da pesquisa do Ibope (2003), com o propósito de detectar qualquer grau de variação entre a amostra selecionada e a do Ibope. Essa comparação permite analisar a validade externa, isto é, a capacidade de generalizar as conclusões dessa pesquisa.

Conforme pesquisa realizada pelo Ibope (2003), os consumidores virtuais formam um seleto grupo que possui renda familiar das classes A e B, grau de instrução com no mínimo o segundo grau, formado majoritariamente por estudantes, jovens e solteiros e tendo realizado compras duas a cinco vezes ao ano com ticket médio de R \$50,00. Em relação a essa pesquisa, a amostra obtida neste estudo demonstrou não ter diferença estatisticamente significativa em todos os itens, exceto em:

- Gênero: na pesquisa Ibope (2003), 63\% dos pesquisados são homens, enquanto na amostra selecionada há uma diferença de apenas I2\% entre 
homens (56\%) e mulheres (44\%). Essa diferença é estatisticamente significativa a um nível de $5 \%$, indicando freqüências distintas. Esse fato vem, no entanto, confirmar os indicadores do aumento da presença da mulher na rede, de acordo com InfoExame (2003).

- Renda familiar: enquanto na pesquisa Ibope 59\% dos usuários de internet estão qualificados para o consumo, com renda mensal familiar situada entre dez e cinqüenta salários mínimos, na amostra selecionada esse valor reduz-se para $52 \%$. Isso demonstra que a renda da amostra selecionada difere, a um nível de 5\% de significância, da renda da pesquisa. Essa diferença pode ser explicada pelo fato de a amostra em questão ter buscado apenas usuários que consomem produtos na internet, diferentemente da pesquisa realizada pelo Ibope (2003), em que foram coletados dados sobre usuários da internet de forma geral.

\section{ANÁLISE DOS DADOS}

A análise dos dados segue o procedimento sugerido por Hair Junior et al. (I998). Primeiro, avaliam-se a coerência das estimativas e o grau de ajustamento do modelo. Em seguida, realiza-se a análise do modelo de mensuração, isto é, o relacionamento entre indicadores e constructos, entre variáveis manifestas e latentes. Finalmente, são reportados os resultados da análise do modelo estrutural e a avaliação das hipóteses utilizadas para testar as relações entre os constructos, e fazem-se as considerações sobre o grau de relacionamento entre estes.

\subsection{ESTIMATIVAS E AJUSTE DO MODELO}

O modelo de equações estruturais pode acomodar ou utilizar para sua análise tanto a matriz de correlação como a de covariância. Para os propósitos da estratégia de confirmação deste trabalho, a matriz de correlação foi utilizada. O primeiro obstáculo na determinação da matriz de correlação foi a presença de variáveis ordinais. A correlação entre variáveis contínuas é medida pelo coeficiente de Pearson. Porém, no caso de correlação entre variáveis ordinais, conforme afirmam Jöreskog e Sörbom (I993), deve ser utilizada a matriz de correlação policlórica/polisserial (polyserial/polychoric correlation). Além disso, é necessário calcular a matriz de covariância assimptótica a ser utilizada como matriz dos pesos das medidas da correlação. Ainda segundo Jöreskog e Sörbom (I993), o modelo deverá ser estimado pelo método dos mínimos quadrados ponderados (MWLS - minimum weight least square), em vez do tradicional método da máxima verossimilhança (MLE - maximum likelihood estimator). 
- CRIAÇÃO E TESTE DE UM MODELO PARA AVALIAÇÃO DE WEBSITES DE COMÉRCIO ELETRÔNICO • LUIZ ANTONIO JOIA • LUIZ CLÁUDIO BARBOSA DE OLIVEIRA

A primeira análise a ser feita é sobre a coerência das estimativas. A maneira correta de identificar problemas desse tipo é a verificação de três itens: variância do erro negativa (também chamada de Heywood case), coeficientes padronizados maiores que o I,o ou erro padrão dos parâmetros muito alto. Após as verificações, concluiu-se que nenhum constructo precisava ser removido do modelo, obtendo-se um modelo estabilizado sem nenhuma offending estimate (problema de estimação) e sem nenhum erro de identificação.

O próximo passo é a avaliação da validade do modelo, feita comumente verificando-se um conjunto de medidas de aderência (goodnes of fit) da Tabela 4 a seguir.

\section{TABELA 4}

RESULTADO DO TESTE DE ADERENCIA DO MODELO FINAL

\begin{tabular}{lclc}
\hline ÍNDICES DE ADERÊNCIA DO MODELO & VALOR & ÍNDICES DE ADERÊNCIA DO MODELO & VALOR \\
\hline Final Fit Function & 0,302 & Normed Fit Index (NFI) & 0,9788 \\
\hline Goodness of Fit Index (GFI) & 0,9246 & Tucker-Lewis Index (TLI) & 0,9962 \\
\hline GFI Adjusted for df (AGFI) & 0,8573 & RMSEA Estimate & 0,05815 \\
\hline Root Mean Square Residual (RMSR) & 0,0526 & RMSEA 90\% Lower Confidence Limit & 0,0331 \\
\hline Chi-Square & 198,39 & RMSEA 90\% Upper Confidence Limit & 0,0832 \\
\hline Chi-Square DF & 170 & Bentler's Comparative Fit Index (CFI) & 0,9351 \\
\hline Pr $>$ Chi-Square & 0,07 & Hoelter's Critical N & 52 \\
\hline
\end{tabular}

Fonte: SAS 8.2.

O modelo está bem ajustado aos dados, visto que os índices NFI, TLI e CFI estão acima do mínimo sugerido de o,9, conforme Klem (I995). Além disso, o AGFI obtido foi de aproximadamente $\circ, 86$, confirmando mais uma vez a boa aderência do modelo, pois quanto mais próximo de I, melhor o modelo. Os resíduos parecem estar pequenos, com o valor do RMSR (Root Mean Square Residual) por volta de $5 \%$, o que confirma o bom ajuste do modelo, segundo Hair Junior et al. (I998). O valor do índice ajustado dos resíduos (RMSEA) deve se situar entre ०,०० (perfect fit) e o,08 (a um nível de 5\% de significância), o que está de acordo com o valor obtido de 0,0582 . O tamanho da amostra também está adequado, visto que é maior que o valor crítico apresentado no índice de Hoelter (HAIR JUNIOR et al., I998). A conclusão da análise desses índices é pela aceitação do modelo como capaz de explicar os dados observados. 
A única medida que contraria todas as outras é a estatística do qui-quadrado. Esse índice é, na verdade, uma medida da falta de aderência (badness offit) do modelo, medindo a variação não explicada dos resíduos. No entanto, conforme afirmam Jöreskog e Sörbom (I993), a medida é calculada com base no tamanho da amostra (N-I vezes o valor mínimo da fit function, onde N é o tamanho da amostra), tendendo a ser superestimada para amostras maiores que duzentas observações. Assim, para evitar esse tipo de problema, é preferível utilizar medidas que compensem o tamanho da amostra, como AGFI, RMSEA, NFI e CFI.

\subsection{MODELO DE MENSURAÇÃO}

A análise do modelo de mensuração inicia-se pela verificação da significância dos indicadore e, em seguida, pela observação da validade da escala. $\mathrm{Na}$ análise do teste t para os coeficientes dos indicadores, para um nível de significância de I\%, todos os parâmetros de mensuração (indicadores) foram considerados estatisticamente significativos em relação aos seus constructos. Uma vez examinada a significância dos indicadores, necessita-se calcular a confiabilidade (reliability) de cada constructo. Um constructo é dito confiável quando seus indicadores explicam a maioria de sua variação.

Três métodos independentes são comumente utilizados para calcular a confiabilidade do constructo: coeficiente alfa de Cronbach, confiabilidade composta (composite) e análise da variância extraída (variance extracted) (SHARMA, I996). A variância extraída é utilizada para verificar a validade discriminante (PEDHAZUR; SCHMELKIN, I99I).

Segundo Hair Junior et al. (I998), o alfa de Cronbach deve ser superior ao mínimo de 0,70, enquanto a confiabilidade composta deve estar acima de o,6o. Outra medida importante para estabelecer o grau de mensuração dos constructos é a variância extraída. Essa medida reflete o grau de dispersão existente entre os indicadores para o determinado constructo. De acordo com Klem (I995), a medida da variância extraída deve estar acima do valor 0,50 para um nível de significância de $5 \%$. As três medidas citadas servem para mensurar o grau de explicação dos indicadores para o constructo. Valores elevados para essas medidas demonstram que o constructo foi bem mensurado (SHARMA, I996). A Tabela 5 demonstra os cálculos do alfa de Cronbach, da confiabilidade composta e da variância extraída para a amostra pesquisada. 
- CRIAÇÃO E TESTE DE UM MODELO PARA AVALIAÇÃO DE WEBSITES DE COMÉRCIO ELETRÔNICO • LUIZ ANTONIO JOIA • LUIZ CLÁUDIO BARBOSA DE OLIVEIRA

\section{TABELA 5}

\section{CONFIABILIDADE DOS INDICADORES}

\begin{tabular}{cccc}
\hline FATOR & ALFA DE CRONBACH & CONFIABILIDADE COMPOSTA & VARIÂNCIA EXTRAÍIA \\
\hline EU & 0,70 & 0,62 & 0,51 \\
\hline OU & 0,77 & 0,70 & 0,58 \\
\hline UT & 0,75 & 0,70 & 0,54 \\
\hline FU & 0,81 & 0,77 & 0,59 \\
\hline PA & 0,89 & 0,84 & 0,69 \\
\hline CR & 0,92 & 0,87 & 0,72 \\
\hline Al & 0,98 & 0,91 & 0,80 \\
\hline
\end{tabular}

Fonte: SAS 8.2.

\subsection{AVALIAÇÃO DAS HIPÓTESES}

Uma vez que os valores de ajustamento do modelo genérico e do modelo de mensuração já tenham sido realizados, pode-se finalmente empreender a análise dos relacionamentos existentes entre os constructos e as hipóteses relacionadas. O seguinte diagrama de caminhos (Figura 2) é utilizado para apresentar os valores encontrados.

\section{FIGURA 2}

DIAGRAMA DE CAMINHOS - COEFICIENTES DA SOLUÇÃO FINAL

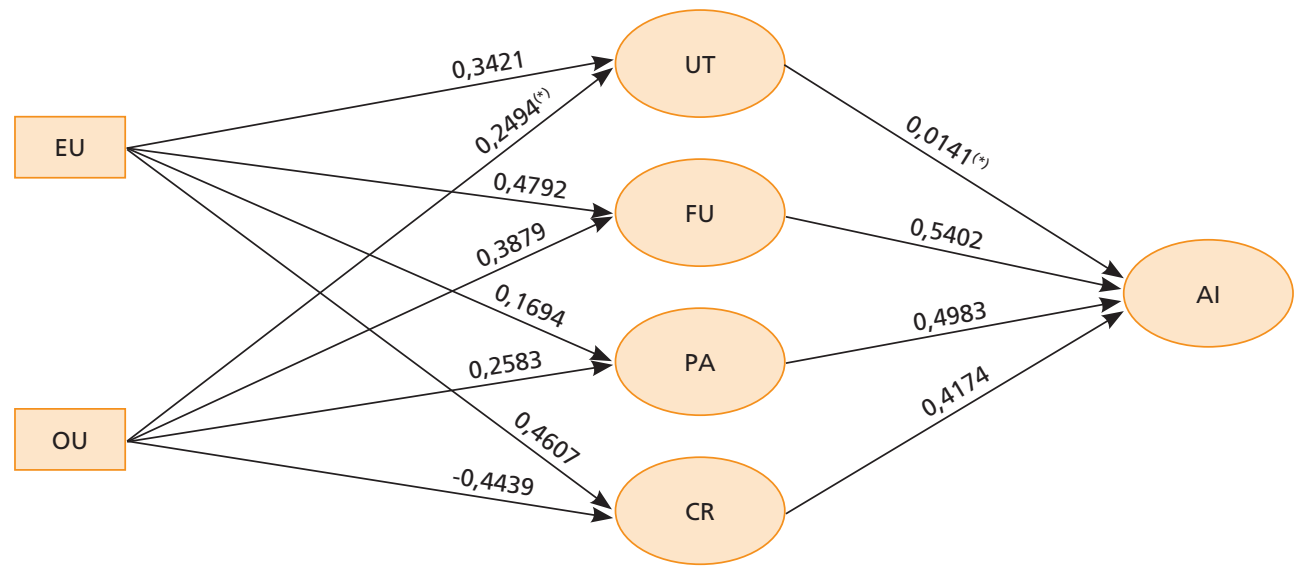

Fonte: SAS 8.2.

(*) Não significativo a I\%. 
Com base nos resultados obtidos pela ferramenta SEM, as hipóteses deste estudo foram testadas (a um nível de significância de i\%) e os resultados obtidos estão descritos na Tabela 6.

\section{TABELA 6}

RESULTADOS DO MODELO ESTRUTURAL

\begin{tabular}{lccccc}
\hline CAMINHO ESTRUTURAL & HIPÓTESE & COEFICIENTES & TESTE T & DECISÃO \\
\hline Estilo usuário $\rightarrow$ utilidade da informação & H1 & 0,3421 & 6,0533 & Aceita \\
\hline Estilo usuário $\rightarrow$ facilidade de uso & H2 & 0,4792 & 8,7907 & Aceita \\
\hline Estilo usuário $\rightarrow$ prazer e atratividade & H3 & 0,1694 & 3,6932 & Aceita \\
\hline Estilo usuário $\rightarrow$ confiança e risco & H4 & 0,4607 & 7,1701 & Aceita \\
\hline Orientação usuário $\rightarrow$ utilidade da informação & H5 & 0,2494 & 2,2857 & Rejeitada \\
\hline Orientação usuário $\rightarrow$ facilidade de uso & H6 & 0,3879 & 4,0621 & Aceita \\
\hline Orientação usuário $\rightarrow$ prazer e atratividade & H7 & 0,2583 & 2,7272 & Aceita \\
\hline Orientação usuário $\rightarrow$ confiança e risco & H8 & $-0,4439$ & $-3,5794$ & Aceita \\
\hline Utilidade da informação $\rightarrow$ atitude e intenção & H9 & $-0,0141$ & 0,1731 & Rejeitada \\
\hline Facilidade de uso $\rightarrow$ atitude e intenção & H10 & 0,5402 & 6,5388 & Aceita \\
\hline Prazer e atratividade $\rightarrow$ atitude e intenção & H11 & 0,4983 & 8,3099 & Aceita \\
\hline Confiança e risco $\rightarrow$ atitude e intenção & H12 & 0,4174 & 10,0312 & Aceita \\
\hline
\end{tabular}

Fonte: SAS 8.2.

Dez dos doze relacionamentos propostos manifestaram significância estatística confirmando as hipóteses apresentadas, e duas hipóteses foram rejeitadas ( $\mathrm{H}_{5}$ e $\left.\mathrm{H}_{9}\right)$.

Conforme a Tabela 4, a orientação do usuário (OU) não foi confirmada como sendo relacionada à percepção da utilidade da informação (UT). Do mesmo modo, a utilidade da informação (UT) não parece afetar a atitude e intenção de compra (AI).

De acordo com o teste realizado, a facilidade de uso, o prazer percebido e a confiança e risco percebidos pelo usuário no website determinam uma atitude positiva, melhorando a atitude e aumentando a intenção de compra de CD via internet. Ou seja, quanto maiores forem a facilidade de uso percebida, o prazer 
percebido e a confiança no website por parte do usuário, melhores serão a sua atitude em relação à compra naquele website e a sua intenção de retornar à loja virtual e comprar.

Com base na análise dos dados realizada e nas informações oriundas do modelo estrutural, pode-se concluir que a loja virtual deve se preocupar em melhorar constantemente seu website para aumentar o tráfego e as vendas. Para conseguir tal objetivo, a pesquisa demonstrou quais indicadores são mais relevantes para cada constructo e o efeito de cada constructo válido dentro do modelo (incluindo a prioridade entre eles), com o propósito de melhorar a atitude e intenção de compra no website de comércio varejista de CD.

\section{CONCLUSÃO}

Com base no referencial teórico apresentado, um modelo de relacionamentos foi proposto e justificado, combinando diversas características presentes em modelos citados anteriormente. Cada componente do modelo foi suportado por um outro já existente, com explicações sobre como as partes se combinam, para formar o modelo completo. Esses elementos foram divididos em aspectos externos e internos do website. Obviamente, este estudo está interessado em avaliar os aspectos presentes em uma loja virtual que melhorem a atitude e aumentem a intenção do usuário em relação à compra de CD. No entanto, conforme explicado, deixar de considerar os aspectos externos do empreendimento via internet compromete a pesquisa, retirando grande parte do poder de explicação do fenômeno. Além disso, as características do consumidor devem ser explicitadas para facilitar o entendimento de seu comportamento.

$\mathrm{Na}$ análise global da aderência do modelo, foi comprovado que o seu uso estava adequado, com um tamanho de amostra bem dimensionado. Os índices calculados (NFI, TLI, AGFI, RMSEA, CFI) indicaram a boa aderência do modelo aos dados obtidos na amostra, inclusive expressando ajustamento superior ao obtido pelo modelo proposto por Heijden (200I). Além disso, o grau de confiabilidade das escalas de medição foi sempre, no mínimo, de o,7 - limite inferior aceitável do alfa de Cronbach -, refletindo o bom ajuste da escala ao conceito mensurado.

Com base na análise de equações estruturais, confirmou-se que o estilo (EU) e a orientação do usuário (OU) são relevantes no entendimento do comportamento do consumidor on-line. Os dados puderam comprovar que a atitude e a intenção de compra do internauta são modificadas pelo modo como este percebe a interface. Conforme informado anteriormente, a facilidade de uso (FU) do website determinou o maior coeficiente de relacionamento com a atitude e intenção 
de compra, com um grau de influência de 54\% de aumento na escala de atitude e intenção (AI) de compra. Outro fator importante na pesquisa é o prazer e a atratividade percebidos (PA), que também influenciam no padrão de compra do consumidor. Esse constructo contribui com um peso de, aproximadamente, $50 \%$ de influência na atitude e intenção (AI) de compra dentro do website. Por último, a relação entre confiança e risco (CR) e atitude e intenção (AI) de compra correspondeu a um grau de influência de $42 \%$. Assim, investir em usabilidade, em layout agradável e prazeroso, e no processo de compra seguro gera uma maior expectativa de vendas no empreendimento, visto que melhora a atitude do consumidor e aumenta a intenção de compra. Além disso, de acordo com o estilo e a orientação do usuário, sua percepção sobre as características do website será diferente.

Somente dois relacionamentos não foram confirmados pelos dados observados, apesar de suportados pela teoria e por outros estudos: entre orientação do usuário (OU) e utilidade percebida (UT); e entre esta última e a atitude e intenção de compra (AI). Apesar de justificadas teoricamente (TURBAN et al., 2000; ZHANG et al., 2000; HEIJDEN, 200I), os dados coletados não confirmaram a existência dessas relações.

Talvez uma mudança recente na característica dos consumidores virtuais que buscam a internet, os quais têm hoje como premissa sempre encontrar informação útil, relevante e de rápido entendimento, tenha tornado esses contructos e seus relacionamentos redundantes para os respondentes. Sugerem-se, no entanto, pesquisas mais aprofundadas em relação a essa questão.

\section{LIMITAÇÕES DO ESTUDO E SUGESTÕES PARA FUTURAS PESQUISAS}

As contribuições proporcionadas por este estudo devem ser avaliadas à luz das limitações existentes no método utilizado. Um obstáculo esteve ligado à definição da amostra. Apesar de ter sido comparada com os resultados obtidos na pesquisa do Ibope (2003), o processo de seleção da amostra não exclui o caráter voluntário/intencional do respondente em participar da pesquisa. Afinal, nem todos os consumidores solicitados a participar realmente o fizeram - foi obtida uma taxa de resposta de 33,07\%. Isso pode levar a conclusões que sejam válidas somente para um perfil de pessoas que não se incomodam em responder a perguntas em questionários on-line.

Ademais, não se pode descartar a relação entre o tipo de produto comercializado e sua propensão em ser vendido por meio eletrônico, conforme provaram Hassanein e Head (2004). Assim, o estudo da atitude e intenção de consumidores virtuais relativamente apenas à compra de CD deve ser considerado, também, um limitador da pesquisa, por ser esse um dos produtos mais homogêneos e previsíveis vendidos na web (HASSANEIN; HEAD, 2004). 
Uma outra limitação do método adotado diz respeito ao uso de atitude e intenção (AI) como um constructo único. Com base nos estudos de Fishbein e Ajzen (apud HEIJDEN, 2000a), esse constructo envolve conceitos teoricamente distintos. No entanto, para efeito de análise via equações estruturais, se divididos em dois, esses constructos não teriam indicadores suficientes para garantir a sua validade discriminante, o que obrigaria a fixação dos erros de medição. Assim, optou-se por medi-los agrupadamente.

Recomenda-se para novas pesquisas refinar a medição desses constructos, realizando-se o mesmo estudo em outros segmentos do comércio eletrônico. Isso permitiria verificar se o mesmo padrão ocorre em outras áreas da economia. Além disso, com novos conceitos teóricos introduzidos no modelo e uma mensuração dos constructos ainda mais refinada, poder-se-ia garantir a aplicabilidade genérica do modelo, estendendo esse tipo de análise a todo o comércio eletrônico.

Uma alteração significativa seria a inclusão de variável dependente relativa à margem de lucro dos websites de comércio eletrônico de $\mathrm{CD}$. Isso permitiria não só validar o modelo vis-à-vis do resultado financeiro obtido, mas também medir o retorno em vendas de CD para cada alteração do aspecto do website. Porém, a dificuldade de conseguir esses dados talvez seja um empecilho a esse tipo de recomendação.

Outro tipo importante de estudo é a análise longitudinal dos parâmetros. Isso demonstraria como os coeficientes do relacionamento entre os conceitos se modificam com o tempo. Segundo Zhang e Dran (200I), algumas características do website vão se alterando com o tempo. Assim, o que hoje é tido como um elemento adicional, no futuro será uma premissa que todas as lojas virtuais devem observar. Portanto, o tipo de análise desenvolvida nesta pesquisa deve ser continuamente refinado. Isso porque sempre há o risco, inerente ao negócio, de mudança no comportamento do consumidor, nas tecnologias de informação e na própria concorrência.

Como última recomendação, os autores sugerem um estudo de controle em empreendimentos virtuais. Controlando as variáveis e realizando modificações na interface, seria possível verificar se o resultado (atitude e intenção de compra) se modifica conforme o esperado/previsto pelo modelo. Desse modo, é possível verificar quantitativamente a relação entre o comportamento passado e a previsão feita pelo modelo.

A análise do comportamento passado e o teste do comportamento futuro são bastante significativos. Criar deliberadamente experimentos in-market (por exemplo, alterando certas características do website) permite que se atribuam diferenças nas respostas a combinações bastante complexas. Criando-se um ciclo rápido de teste-análise, é possível adaptar constantemente o que é testado ao que é aprendido pela organização. O aprendizado contínuo feito dessa maneira 
oferece dois benefícios: fornece rápido feedback do mercado, o que permite que a empresa detecte mudança nos comportamentos dos clientes e efeito das ações dos concorrentes; e revela novas formas de gerar valor econômico por meio da mudança da experiência do consumidor, passando de ações pontuais para a geração de valor sustentável. No entanto, esse tipo de coordenação e cooperação para a análise exige um grau de integração normalmente ainda difícil de ser conseguido nas empresas.

\section{REFERÊTNCIAS}

BELLMAN, S.; LOHSE, G. L.; JOHNSON, E. J. Predictors of online buying Behavior. Communications of $A C M$, v. 42. n. 12, p. 32-38, Dec. I999.

BRONDMO, H. P. The engaged customer: the new rules of Internet direct marketing. New York: Harper Business, 200I.

DAVIS, F. D. Perceived usefulness, perceived ease of use, and user acceptance of information technology. MIS Quarterly, v. I3, n. 2, p. 319-340, Sept. I989.

GODIN, S. Unleashing the idea virus: stop marketing at people! Turn your ideas into epidemics by helping your customers do the marketing thing for you. New York: Hyperion, 200I.

GREENWALD, A. R.; KEPHART, J. O. Shopbots and pricebots. In: THE PROCEEDINGS OF INTERNATIONAL JOINT CONFERENCE ON ARTIFICIAL INTELLIGENCE. Stockholm, I999. HAIR JUNIOR, J. F. et al. Multivariate data analysis. 5. ed. New Jersey: Prentice Hall, I998.

HANSON, W. Principles of internet marketing. Cincinnati: South-Western College Publishing, 2000.

HASSANEIN, K.; HEAD, M. The influence of product type on online trust. In: $\mathrm{I7}^{\mathrm{TH}}$ ELECTRONIC COMMERCE CONFERENCE, 2004, Bled, Slovenia. Proceedings... Bled, Slovenia, 2004.

HEIJDEN, H. van der. E-TAM - a revision of the technology acceptance model to explain website revisits. Research Memorandum, v. 29, Sept. 2000a.

Using the technology acceptance model to predict website usage: extension and emprirical test. Research Memorandum, v. 25, July 2000 b.

Factors influencing the usage of websites: the case of a Generic Portal in the Netherlands.

In: $4^{\text {TH }}$ BLED ELECTRONIC COMMERCE CONFERENCE: E-EVERYTHING: E-COMMERCE, E-GOVERNMENT, E-HOUSEHOLD, E-DEMOCRACY, 200I, Bled, Slovenia. Proceedings... Bled, Slovenia, 200I.

HOFFMAN, D.; BATESON, J. E. G. Essentials of services marketing. London: International Thomson Publishing, I999.

HOFFMAN, D. L.; NOVAK, T. P. Marketing in hypermedia computer mediated environment: conceptual foundations. Owen Graduate School of Management and Vanderbilt University. I995. Disponível em: <http://www2000.ogsm.vanderbilt.edu>. Acesso em: 2I mar. 2005.

HOFFMAN, D. L.; NOVAK, T. P.; PERALTA, M. Building consumer trust in online environments: The case for information privacy. 1998. Owen Graduate School of Management and Vanderbilt University. Disponível em: <http://www2000.ogsm.vanderbilt.edu>. Acesso em: I2 nov. 2004. 
- CRIAÇÃO E TESTE DE UM MODELO PARA AVALIAÇÃO DE WEBSITES DE COMÉRCIO ELETRÔNICO • LUIZ ANTONIO JOIA • LUIZ CLÁUDIO BARBOSA DE OLIVEIRA

IBOPE. $8^{\circ}$ Pesquisa Internet Brasil - Ibope. 2003. Disponível em: <http://www.ibope.com.br>. Acesso em: 5 maio 2005 .

INFOEXAME. Plantão INFO - 429 milhões de pessoas estão online no mundo. Disponível em: <http:// www2.uol.com.br/info/index.shl>. Acesso em: I2 maio 2003.

JARVENPAA, S. L.; TRACTINSKY, N.; VITALE, M. Consumer trust in an internet store. Information Technology \& Management, v. I, n. I, p. 45-7I, 2000.

JÖRESKOG, K. G.; SÖRBOM, D. Lisrel 8: structural equation modeling with Simplis command language. Mooresville: Scientific Software, I993.

KIM, D. J.; CHO, B.; RAO, H. R. Effects of consumer lifestyles on purchasing behavior on the internet: a conceptual framework and empirical validation. In: TWENTY FIRST INTERNATIONAL CONFERENCE ON INFORMATION SYSTEMS, 2000, Brisbane, Australia. Proceedings... Brisbane, Australia, 2000. p. 688-695.

KLEM, L. Path analysis: reading and understanding multivariate statistics. In: GRIMM, L. G.; YARNOLD, P. R. (Ed.). Reading and understanding multivariate statistics. Washington: American Psychological Association, I995.

KOTLER, P.; ARMSTRONG, G. Princípios de marketing. 7. ed. Rio de Janeiro: LTC, I999.

LEDERED, A. L. et al. The technology acceptance model and the world wide web. Conference of Decision Support Systems, v. 29, p. 269-282, 2000.

LOEBBECKE, C.; HORNIG, V. Increasing trust in e-commerce: concepts and examples of insurance solutions. In: $\mathrm{I}_{4}^{\mathrm{TH}}$ BLED ELECTRONIC COMMERCE CONFERENCE, 2OoI, Bled, Slovenia. Proceedings... Bled, Slovenia, $200 \mathrm{I}$.

LOHSE, G. L.; SPILLER, P. Electronic shopping: the effect of customer interface on traffic and sales. Communications of ACM, v. 4I, n. 7, p. 8I-87, I999.

MOE, W. W.; FADER, P. S. Modeling online store visit patterns as a measure of customer satisfaction. 200I. Disponível em: <http://2I6.I07.I3I.I55/archive/papers/>. Acesso em: 2 mar. 2005.

NIELSEN, J. Projetando web sites - Design web usability. 2 ed. Rio de Janeiro: Campus, 200 I.

PARENTE, J. Varejo no Brasil. São Paulo: Atlas, 2000.

PEDHAZUR, E.; SCHMELKIN, L. P. Measurement, design and analysis: an Integrated approach. Hillsdale: Lawrence Erlbaum Associates, I99I.

REEDY, J.; SCHULLO, S.; ZIMMERMAN, K. Electronic marketing: integrating electronic resources into the marketing process. Cincinnati: South-Western, 200I.

RUST, R. T.; ZEITHAML, V. A.; LEMON, K. N. Driving customer equity: how customer lifetime value is reshaping corporate strategy. New York: The Free Press, 200I.

SCHUBERT, P.; SELZ, D. Measuring the effectiveness of electronic commerce sites going beyond traditional marketing paradigms. In: $32^{\mathrm{ND}}$ HAWAII INTERNATIONAL CONFERENCE ON SYSTEM SCIENCES, I999, Hawaii. Proceedings... Hawaii, I999.

SHARMA, S. Applied Multivariate Techniques. New York : John Willey \& Sons, I996.

SMITH, M. D.; BAILEY, J.; BRYNJOLFSSON, E. Understanding digital markets: review and assessment. I999. Disponível em: <http://www.ecommerce.mit.edu/papers/ude>. Acesso em: jul. 200I.

SNEDECOR, G. W.; COCHRAN, W. G. Statistical methods. Iowa: Iowa State University Press, I989.

TAYLOR, H. Does Internet research work? Journal of the Market Research Society, London, I9992000. 
TROCHIM, W. M. K. The research methods knowledge base. 2. ed. Disponível em: <http://www. trochim.human.cornell.edu/kb/index.htm>. Acesso em: mar. 200I.

TURBAN, E.; GEHRKE, D. Determinants of successful website design: relative importance and recommendations for effectiveness. In: $32^{\text {ND }}$ HAWAII INTERNATIONAL CONFERENCE ON SYSTEM SCIENCES, I999, Hawaii. Proceedings... Hawaii, I999.

TURBAN, E. et al. Electronic commerce - a managerial perspective. Rio de Janeiro: Prentice Hall do Brasil, 2000.

URBAN, G. L.; SULTAN, F.; QUALLS, W. J. Placing trust at the center of your internet strategy. Sloan Management Review, Fall 2000.

VENKATESH, V. Determinants of perceived ease of use: integration control, intrinsic motivation and emotion into the technology acceptance model. 2000. Disponível em: <http://isr.commerce.ubc. ca/Abstract/II-4-Venkatesh.html>. Acesso em: set. 2002.

WOLFINBARGER, M.; GILLY, M. Shopping online for freedom, control and fun. California: California State University Long Beach, College of Business Administration, 200I.

WONNACOTT, T.; WONNACOTT, R. Introductory statistics for business and economics. 2. ed. New York: John Wiley \& Sons, I972.

ZHANG, P.; DRAN, G. von. Expectations and rankings of website quality features: results of two studies on user perceptions In: $34^{\text {TH }}$ HAWAII INTERNATIONAL CONFERENCE ON SYSTEM SCIENCES, 200I, Hawaii. Proceedings... Hawaii, 200 I.

ZHANG, P. et al. A two factor theory for website design. In: $33^{\text {TH }}$ HAWAII INTERNATIONAL CONFERENCE ON SYSTEM SCIENCES, 2000, Hawaii. Proceedings... Hawaii, 2000.

\section{TRAMITAÇ ÃO}

Recebido em 2/8/2006

Aprovado em 5/3/2007 